Article

\title{
Assessment, Validation and Application to Real Samples of an RP-HPLC Method for the Determination of Guayulins A, B, C and D in Guayule Shrub
}

\author{
Nadia Spano ${ }^{1, *}$, Paola Meloni ${ }^{1}$, Ilenia Idda ${ }^{1}$, Alberto Mariani ${ }^{1}$, Maria Itria Pilo ${ }^{1}$ (i), \\ Valeria Marina Nurchi ${ }^{2}{ }^{(1)}$, Joanna Izabela Lachowicz ${ }^{2}$, Ernesto Rivera ${ }^{3}$, \\ Ancelmo Orona-Espino ${ }^{4}$ and Gavino Sanna ${ }^{1, * \text { (D) }}$ \\ 1 Dipartimento di Chimica e Farmacia, Università degli Studi di Sassari, via Vienna 2, 07100 Sassari, Italy; \\ paola94meloni@gmail.com (P.M.); ilenia.idda@gmail.com (I.I.); mariani@uniss.it (A.M.); \\ mpilo@uniss.it (M.I.P.) \\ 2 Dipartimento di Scienze Chimiche e Geologiche, Cittadella Universitaria di Monserrato, \\ 09042 Monserrato, Italy; nurchi@unica.it (V.M.N.); lachowicz@unica.it (J.I.L.) \\ 3 Instituto de Investigaciones en Materiales UNAM, Circuito Exterior Ciudad Universitaria, CP 04510 Mexico \\ DF, Mexico; riverage@unam.mx \\ 4 Facultad de Ciencias Biológicas, Universidad Juárez del Estado de Durango, 35010 Gómez Palacio, Mexico; \\ chemo74@hotmail.com \\ * Correspondence: nspano@uniss.it (N.S.); sanna@uniss.it (G.S.); Tel.: +39-079-229569 (N.S.); \\ +39-079-229500 (G.S.); Fax: +39-079-0971388 (G.S.)
}

Received: 25 February 2018; Accepted: 22 March 2018; Published: 9 April 2018

\begin{abstract}
Guayule (Parthenium argentatum Gray) is a shrub native to the arid regions of Mexico. In the last decades, significant attention to its cultivation has arisen because it is the raw material for the production of hypoallergenic natural rubber. Guayule biomass also contains high amounts of resin, which is not normally exploited in any way. Among other sesquiterpenic esters, guayulins (i.e., the parteniol esters of cinnamic acid, guayulin A, or of anisic acid, guayulin B) are contained in resin. In addition, minor amounts of guayulin $\mathrm{C}$ and guayulin $\mathrm{D}$ are formed by degradation/oxidation of guayulins A and B, respectively. Guayulins likely act as cinnamate and $p$-anisate reservoirs for the Guayule shrub; in addition, it has been postulated that they might have a key role in the chemical defense system of Guayule. Furthermore, it seems reasonable that guayulins may possess significant biological properties (e.g., antibacterial and anticancer activities), in close analogy with those shown by sesquiterpene lactones contained in many other species of Parthenum genus. As a matter of fact, guayulins A and B play an important role in the synthesis of antineoplastics used in breast cancer treatment. In this contribution we propose an original and validated RP-HPLC approach to the simultaneous quantification of guayulins A, B, C and D. The procedure of resin extraction from Guayule biomass has been optimized in terms of both extraction method and solvent. RP-HPLC separation has been accomplished by an Ascentis ${ }^{\circledR}$ C18 column under isocratic elution with a 80:20 (v:v) acetonitrile:water mixture. Validation was carried out in terms of limits of detection and quantification, linearity, precision, and trueness. Finally, the method was tested with a number of fresh and seasoned samples of spontaneous Guayule shrub from Mexico.
\end{abstract}

Keywords: guayule; Parthenium argentatum Gray; resin; guayulins; RP-HPLC 


\section{Introduction}

Guayule (Parthenium argentatum Gray) is a perennial and low-growing shrub belonging to the Asteraceae family, native to arid regions of Mexico and the southwestern United States. Since the early years of last century it has gained considerable industrial and scientific interest for the production of a kind of natural rubber exhibiting properties similar to that obtained from the Hevea brasiliensis tree $[1,2]$. In recent decades, the discovery that guayule latex is free from the allergenic proteins contained in the Hevea latex led to a reemphasis and expansion of guayule research, mainly aimed at the production of hygiene and medical products compliant with the strictest standards of quality for hypoallergenic rubber [3]. Together with rubber, Guayule plant also contains a resinous material (the so-called 'resin'), which has to be separated from rubber in order not to compromise its physical and technological properties. Rubber ( $8-26 \%$ of dry weight) and resin (5-25\% of dry weight) are present in amounts roughly equivalent in Guayule shrub [1]. Since the resin fraction is soluble in polar solvents (like acetone), whereas the rubber one can be dissolved only in non-polar solvents (like hexane), sequential or simultaneous extractions have to be made in order to separate these fractions from plant tissue and, eventually, the resin from the latex [1]. The high manufacturing costs of the process of rubber extraction from Guayule make the success of its exploitation dependent to a large extent on the possibility of using as much as possible the residual plant fractions, like resin or bagasse, as raw materials to obtain high-value coproducts [4].

Guayule resin is usually obtained from the extraction by the ground whole shrub or the coagulated latex with polar solvents, usually acetone [5]. Despite the fact that it is often not valorized and hence discarded, resin is a very interesting fraction from both an academic and an industrial point of view. Indeed, it is not only rich in sesquiterpenoids and triterpenes of potential commercial value [6], it also contains a variety of interesting secondary metabolites of the shrub, like guayulins [6-8]. Guayulins A ((1R,2S,4Z,8Z,10S)-4,8,11,11-tetramethylbicyclo[8.1.0]undeca-4,8-dien-2-yl(2E)-3-phenylprop-2-enoate) and B ((4Z,8Z)-4,8,11,11-tetramethylbicyclo[8.1.0]undeca-4,8-dien-2-yl 4-methoxybenzoate) are respectively the cinnamic and anisic esters of the partheniol ((1S,2E,6E,9S,10R)-3,7,11,11tetramethylbicyclo[8.1.0]undeca-2,6-dien-9-ol) [7], whereas the guayulin C ((1AR,1BR,2R, 4AR,7S,7AR)-2-hydroxy-1,1,2-trimethyl-5-methylidene-decahydro-1H- cyclopropa [E]azulen-7-yl (2E)-3-phenylprop-2-enoate) and guayulin D ((1AR,1BR,2R,4AR,7S,7AR)-2-hydroxy-1,1,2-trimethyl-5methylidene-decahydro-1H-cyclopropa[E]azulen-7-yl 4-methoxybenzoate) are likely formed by the oxidation of the guayulines A and B, respectively $[6,8]$. Scheme 1 reports the structures of these molecules.

(a)<smiles>[R]C(C/C(C)=C/CCC(C)=CC1CC1C1CC1(C)C)C1CC1</smiles>

$\mathbf{R}=t$-cinnamate, guayuline $\mathrm{A}$

$\mathbf{R}=p$-anisate, guayuline $\mathrm{B}$ (b)

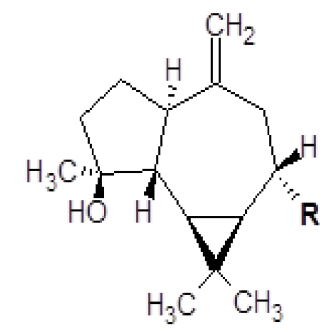

$\mathbf{R}=t$-cinnamate, guayuline $C$

$\mathbf{R}=p$-anisate, guayuline $\mathrm{D}$

Scheme 1. Structures of guayulins A and B (a) and guayulins C and D (b).

Guayulins A and B are abundant in the resin. Namely, up to $10 \%$ of the resin mass is constituted of guayulin A [9-11], while the relevant amount of guayulin B in the resin from India is between $0.1 \%$ and 3\% [10]. The wide variability in the amounts of guayulins $\mathrm{A}$ and $\mathrm{B}$ in the resin depends on a number of factors, such as: soil nature, climate, phenological phases, cultivar, age and, mainly, the nature of the 
different parts of the shrub $[10,11]$. On the other hand, the concentrations of guayulins $C$ and $D$ in resin seems to be much lower than those of guayulins A and B. Schloman et al. estimated that the amounts of guayulins $C$ and $D$ in resin are between $50 \%$ and less than $10 \%$ of the amount of the guayulin $A$ and $B$, respectively [6]. Guayulins likely act as cinnamate and $p$-anisate reservoirs for the Guayule shrub: when it is required by the plant, a metabolic turnover releases the corresponding free acid [4]. The hypothesis that guayulins and rubber may have a common biochemical link [12] suggested Teetor et al. [11] to use the amounts of guayulins A and B as possible predictive tools in order to evaluate the rubber content in Guayule shrub, but the results obtained were absolutely unsatisfactory. It is noteworthy that, in Heliantheae tribe and Parthenium genus, only Parthenium argentatum contains both guayulins and rubber, whereas it does not contain any sesquiterpene lactone (i.e., one of the principal families of compounds easily found in almost all the other species of Parthenum genus) [13]. Since the sesquiterpene lactones are synthesized in the Parthenum genus plants as chemical defense agents against animal attacks (these molecules are toxic for livestock, a deterrent for insects and cause severe contact allergies in mammalians) [14], it seems reasonable that guayulins might play a similar physiological role also in Parthenium argentatum. Results of pioneering studies conducted by Rodriguez et al. supported this insight [15]. As a matter of fact, guayulin A has been found to be a powerful contact allergen for guinea pigs [8] while the same behavior has not been observed for guayulin B. For humans, the sensitizing power of guayulin A is much smaller than for laboratory animals. Hence, the possibility that traces of guayulin A may be present in the guayule rubber at a sensitizing level seems to be remote at the moment [16]. In analogy to what was observed for sesquiterpene lactones in most of the species of Parthenum genus, it is likely that guayulins may also exhibit other significant biological properties (i.e., antibacterial and anticancer activities) [15]. Indeed, guayulines A and B act as biological triggers in the synthesis of lychnostatine and paclitaxel, which are antineoplastic agents used in breast cancer treatment.

It is evident that the continuous rise of interest in the properties of guayulins needs the development of reliable, accurate and sensitive analytical methods for their identification and quantification. Until now, the analytical methods reported in literature for the measurement of the amount of guayulins in Guayule resin [3,4,8,11,13,17-19] or latex [16] are scarce and all lacking of any validation protocol. Obviously, chromatographic methods are dominant in the analytical characterization of such analytes and, among them, HPLC is the preferred approach. While few papers reported the presence of guayulins $C$ and $D$ in extracts from resin $[6,8,11]$, the quantification of these analytes has been never simultaneously carried out to that of guayulins A and B [6,8]. Hence, it is evident that at present a validated HPLC procedure aimed to simultaneously quantify the amounts of all known guayulins is not reported in literature. For this reason, the principal aim of this contribution is to develop, validate and test with real samples an original RP-HPLC method devoted to the quantification of guayulins A, B, C and D in different parts of Guayule shrub from Mexico. As a side result of this study, different approaches of extraction of analytes from the matrix were also compared in order to increase efficiency and make the procedure more eco-friendly.

\section{Materials and Methods}

\subsection{Samples and Sample Preparation}

Spontaneous Guayule was collected in the province of San Pedro del Gallo, Durango, Mexico, along the highways where it naturally grows. All the harvested Guayule shrubs were about $60 \mathrm{~cm}$ high, thus estimated to be between 15 and 18 year old [20]. These plants were harvested in 2015 in two sites located in the Durango desert. The first one (sampling of January 2015) is in the Mexican Federal Highway 30 (road section Bermejillo-El Palmito, GPS coordinates: $25^{\circ} 52^{\prime} 20.68^{\prime \prime}$ N, $104^{\circ} 06^{\prime} 26.07^{\prime \prime}$ W), whereas the second one (sampling of September 2015) is along the highway Paso Nacional-San Pedro del Gallo (GPS coordinates: $25^{\circ} 40^{\prime} 50.41^{\prime \prime} \mathrm{N}, 104^{\circ} 16^{\prime} 33.53^{\prime \prime} \mathrm{W}$ ). A total of twenty plants (ten for each sampling) were harvested following the recommendations specified by the Official Mexican Rule [21], which establishes the procedures for the sampling, transportation and storage. Shrubs collected in the 
harvest of January 2015 were air-dried at room temperature in open air for eight months, whereas shrubs collected in the harvest of September 2015 did not undergo any drying process. Only a few millimeters of rain fell during the first harvest. Just arrived in the laboratory, all shrubs were cut up to constitute three fractions: leaves (L), fine stems (less than $10 \mathrm{~mm}$ in diameter, FS) and coarse stems (more than $10 \mathrm{~mm}$ of diameter, CS). All fractions were stored in a freezer at $-18{ }^{\circ} \mathrm{C}$ until sample preparation. Before extraction, samples were allowed to reach the room temperature. Later, all samples were dried at $50{ }^{\circ} \mathrm{C}$ until reaching constant weight, roughly pieced and then ground.

\subsection{Chemicals and Reagents}

All reagents were at least of analytical grade. Ethanol (assay $\geq 99.8 \%)$, acetone $(\geq 99.8 \%)$ and acetonitrile ( $\geq 99.9 \%$ ) were purchased from Sigma-Aldrich (Milan, Italy), whereas ultrapure (Type 1) water (specific resistance $18 \mathrm{M} \Omega$ ) was used throughout the analyses. Guayulins A, B, C and D were from 1717 CheMall (Mundelein, IL, USA).

\subsection{Instrumentation}

Ultrapure water was from a New Human Power II Scholar UV apparatus (Human Corporation, Seoul, Korea). A Retsch Grindomix GM 200 knife mill (Verder Scientific, Torre Boldone, Italy) was used to grind the samples before extraction. ASE extractions were performed by an Accelerated Solvent Extractor Dionex ASE 200, equipped with a Dionex Solvent Controller System (Dionex Corporation, Sunnyvale, CA, USA), whereas ultrasonic assisted extractions were performed by a Bandelin-Sonorex model RK $255 \mathrm{H}$ ultrasonic bath. Solvent was evaporated by extracted samples by a Büchi Rotovapor model EL130 (Büchi, Cornaredo, Italy). The HPLC equipment consisted of a Series 200 binary pump, a sampling valve, a $20 \mu \mathrm{L}$ sample loop, a Series 200 LC column oven, and a Series $200 \mathrm{UV}$-Vis variable wavelength detector, all from PerkinElmer, Milan, Italy. Data were processed using a Turbochrom Workstation Software (PerkinElmer, Milan, Italy). Before use, the mobile phase was filtered through a $0.45 \mu \mathrm{m}$ membrane from Millipore (Bedford, MA, USA) to remove any particulates.

\subsection{Validation}

Validation of the proposed method was accomplished on the basis of limit of detection (LoD), limit of quantification (LoQ), linearity, precision (measured as both repeatability and intermediate precision) and trueness (measured by recovery tests of each analyte on real Guayule samples). LoD was calculated according to the Upper Limit Approach (ULA1) approved by IUPAC [22]. For each analyte, four different solutions at increasing concentrations not far from the expected LoD (i.e., between 0.02 and $0.1 \mathrm{mg} \mathrm{L}^{-1}$ for each analyte) were prepared and analyzed. Each measurement was performed in triplicate. In addition, the ULA1 approach recommends that the LoQ value is three times the relevant LoD value. Linearity was checked on at least three orders of magnitude of concentration, as a function of the relative abundance of each guayulin in the different parts of the Guayule shrub. Precision was evaluated in terms of both repeatability (i.e., the CV measured in five consecutive replicates of the same sample in the same analytical session) and intermediate precision (i.e., the $\mathrm{CV}$ obtained in five consecutive replicates of the same sample in different analytical sessions across two weeks). The acceptability of these precision scores was checked in terms of HorRat ratio values (i.e., the ratio between experimental and theoretical CV measured on the basis of Horwitz's theory) [23]. Due to the lack of any certified reference materials or of reliable independent analytical methods, trueness was estimated through recovery tests. Four aliquots of the acetone (or ethanol) extract of the stems were submitted to the overall analytical procedure after the addition of increasing amounts of each guayulins to three of them. For each analyte, a plot of analytical concentration versus the added amounts of mass was obtained: the recovery is represented by the percent slope value of the regression line. The estimation of bias was made in duplicate. Acceptability of the trueness values has been established according to the guidelines described in the AOAC manual for peer-verified methods [24]. 


\section{Results}

\subsection{Choice of the Extraction Procedure}

The choice of extraction technique of resin by Guayule biomass (i.e., leaves, fine stems, coarse stems) was accomplished by means of a preliminary comparison among three well-known procedures: the traditional extraction with warm solvents $\left(40{ }^{\circ} \mathrm{C}\right)$ (WSE), the ultrasonic-assisted extraction (UAE) and the accelerated solvent extraction (ASE). Acetone (i.e., the most used solvent for the extraction of the resin from Guayule biomass) and ethanol, one of the most important green polar solvent, were the pure solvents used in this phase. Quantification of resin in organic extracts was performed by means of UV-Vis spectroscopy according to literature methods [25].

\subsubsection{Procedures of Extraction}

\section{Extraction with Warm Solvents (WSE)}

Firstly, $2.5 \mathrm{~g}$ of selected Guayule biomass were suspended in $15 \mathrm{~mL}$ of acetone (or ethanol). The mixture was stirred and heated up to $40{ }^{\circ} \mathrm{C}$ in a thermostatic bath for $20 \mathrm{~min}$. After the separation of the solvent from the biomass and its replacement with a new $15 \mathrm{~mL}$ aliquot, the extraction procedure was repeated for an additional two times. The three extracts were collected and made up to the final volume of $50 \mathrm{~mL}$ with pure solvent.

\section{Ultrasonic Assisted Extraction (UAE)}

Firstly, $2.5 \mathrm{~g}$ of selected Guayule biomass were suspended in $15 \mathrm{~mL}$ of acetone (or ethanol). The mixture was stirred at room temperature in an ultrasonic bath for $20 \mathrm{~min}$. After the separation of the solvent from the biomass and its replacement with a new $15 \mathrm{~mL}$ aliquot, the extraction procedure was repeated for an additional two times. The three extracts were collected and made up to the final volume of $50 \mathrm{~mL}$ with pure solvent.

\section{Accelerated Solvent Extraction (ASE)}

Firstly, $5.0 \mathrm{~g}$ of selected Guayule biomass were transferred in a $22 \mathrm{~mL}$ thimble. Extraction was performed using $20 \mathrm{~mL}$ of the chosen solvent (acetone or ethanol). The extraction temperature was set at $40{ }^{\circ} \mathrm{C}$. Three consecutive extraction cycles have been performed for each biomass aliquot. The organic extracts were joined and made up to $100 \mathrm{~mL}$ with pure solvent.

Figure 1 reports a comparison of resin yields (\% on the initial biomass amount) measured for each extraction method and each biomass analyzed.

Data obtained substantiate that the UAE method is characterized by the least recovery in resin, whereas the performances of WSE and ASE methods are roughly comparable as a function of the nature of Guayule biomass and the extraction solvent. However, it is possible to note that the net amount of resin extracted from the fine stems by means of ASE method is statistically higher than that obtained by WSE, whereas the yields in resin measured in the extraction from coarse stems are not statistically different among them from both WSE and ASE methods. Keeping into account the better intrinsic reproducibility of the ASE approach as compared with the WSE method, the ASE extraction method with acetone and ethanol was chosen as the technique used of the present analytical method. 

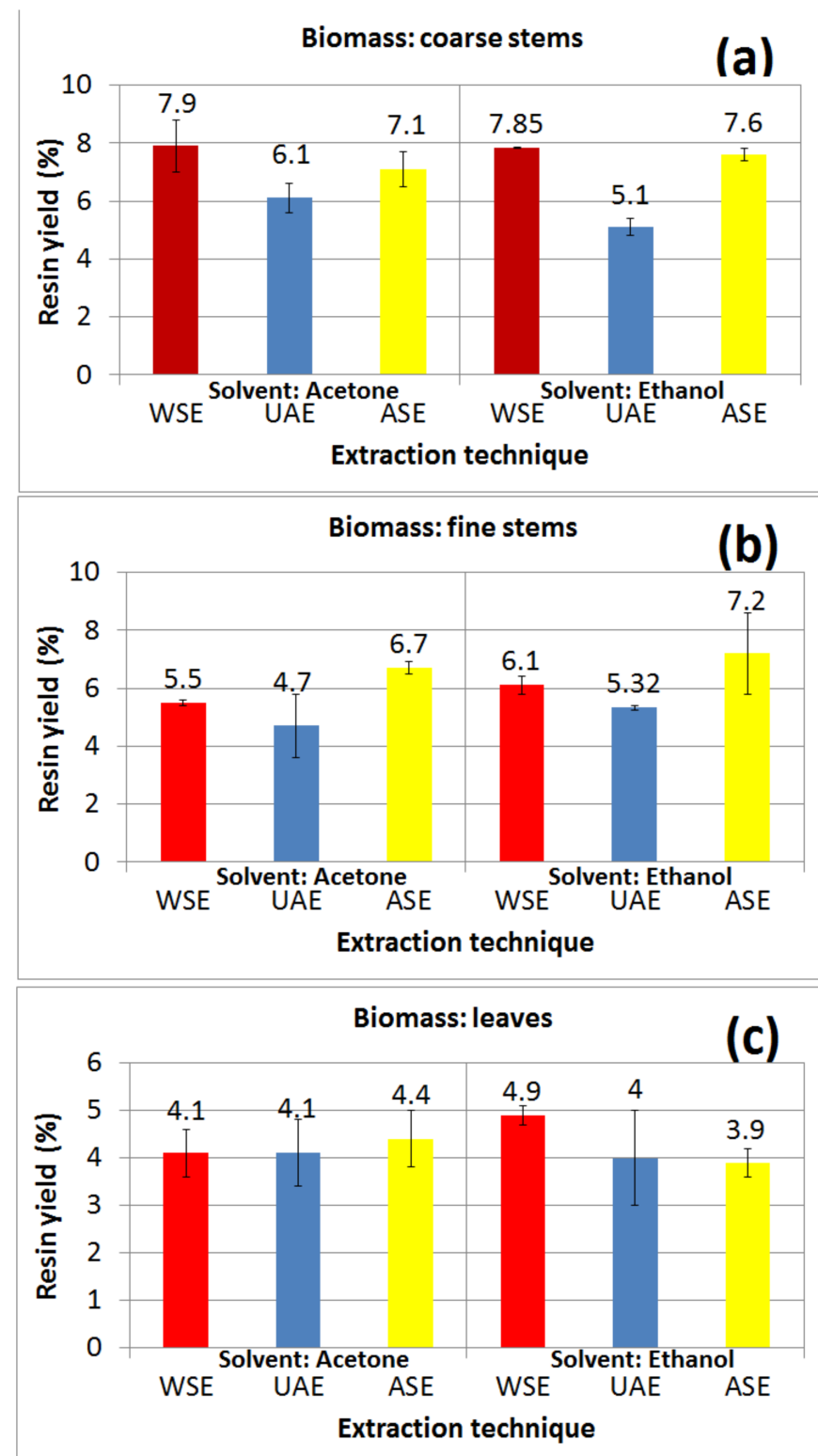

Figure 1. Comparison of average solvent extraction yields in resin ( $\%$ on the biomass amount), $n=3$. Solvents: acetone or ethanol. (a) biomass: coarse stems; (b) biomass: fine stems; (c) biomass: leaves. WSE: warm solvent extraction; UAE: ultrasonic-assisted extraction; ASE: accelerated solvent extraction.

\subsection{Assessment of the Chromatographic Method}

The first RP-HPLC methods aimed to quantify guayulins in Guayule resin were developed more than thirty-five years ago, and were only aimed at the determination of guayulins A and B. On the other hand, reliable RP-HPLC methods devoted to measure the concentration of guayulins C and D are still absent in the literature. For the reader's convenience, Table 1 summarizes the key features of different literature RP-HPLC methods for the determination of guayulins.

Furthermore, it is of utmost significance to underline that no analytical method proposed until now has been validated, and this poses serious doubts about the overall reliability of the data reported. The results of preliminary tests performed on selected literature methods suggested the assessment of an original procedure, based on a more recent C18 stationary phase than those reported in Table 1 . Ascentis ${ }^{\circledR} \mathrm{C} 18$ is a monomeric-type, fourth-generation, octadecylsiloxane-bonded silica stationary phase, introduced in the market in the early 2000s, and characterized by unusually high surface area 
$\left(450 \mathrm{~m}^{2} \mathrm{~g}^{-1}\right)$ if compared with other $\mathrm{C} 18$ phases having similar porosity $(10 \mu \mathrm{m})$ and surface coverage $\left(3.7 \mu \mathrm{mol} \mathrm{m}{ }^{-2}\right)$. Since Ascentis ${ }^{\circledR}$ stationary phases have already been successfully used for the HPLC determination of terpenoids in vegetal matrices [26], we considered useful to check the performances of an Ascentis ${ }^{\circledR} \mathrm{C} 18(25 \mathrm{~cm} \times 4.6 \mathrm{~mm} \times 5 \mu \mathrm{m})$ column in the separation of guayulins A, B, C and D. Further steps of the method assessment have been devoted to optimize the composition of the mobile phase and to the choice of the best wavelength of UV detection. Starting from the literature methods, different methanol:water and acetonitrile:water mixtures have been tested, working both in isocratic and in gradient elution. An acetonitrile:water 80:20 (v:v) solution provided the best results. In addition, the acquisition of chromatograms in the wavelength range between $250 \mathrm{~nm}$ and $280 \mathrm{~nm}$ demonstrates that the best compromise among different UV maximum absorptions of the analytes is the $\lambda$ at $262 \mathrm{~nm}$, as previously reported in the literature $[4,6,10,11]$. Table 2 summarizes the operating conditions of the chromatographic method proposed.

Table 1. Key features of selected literature RP-HPLC methods of analysis for guayulins in resin extracts from Guayule.

\begin{tabular}{|c|c|c|c|c|c|}
\hline Stationary Phase $(\mathrm{mm} \times \mathrm{mm} \times \mu \mathrm{m})$ & Mobile Phase $(v: v)$ & Flow Rate $\left(\mathrm{mL} \mathrm{min}{ }^{-1}\right)$ & $\lambda$ of Quantification $(\mathrm{nm})$ & Guayulins Quantified & Reference \\
\hline MicroPak MCH-10 $(300 \times 4.6$ × 10) & $\begin{array}{l}\text { from } \mathrm{CH}_{3} \mathrm{CN}: \mathrm{H}_{2} \mathrm{O} 50: 50 \text { to } \\
\qquad \mathrm{CH}_{3} \mathrm{CN}: \mathrm{H}_{2} \mathrm{O} 75: 25\end{array}$ & n.r. & 262 & $\mathrm{~A}$ and $\mathrm{B}^{1}$ & [6] \\
\hline C18 column $^{2}$ & $\mathrm{MeOH}: \mathrm{H}_{2} \mathrm{O}$ gradient elution ${ }^{2}$ & n.r. & 215 & C and D & [8] \\
\hline RP-C18 column ${ }^{2}$ & $\mathrm{MeOH}: \mathrm{H}_{2} \mathrm{O} 89: 11$ & 1 & 254 & $A$ and $B$ & [10] \\
\hline Microsorb-MV $(250$ × $4.6 \times 5)$ & $\begin{array}{c}\text { from } \mathrm{CH}_{3} \mathrm{CN}: \mathrm{H}_{2} \mathrm{O} 80: 20 \text { to } \\
\text { pure } \mathrm{CH}_{3} \mathrm{CN}\end{array}$ & 1.5 & 262 & $\mathrm{~A}$ and $\mathrm{B}^{3}$ & {$[11,16,17,19]$} \\
\hline $\begin{array}{l}\text { Altex Ultrasphere ODS type } \\
(150 \times 4.6 \times 5)\end{array}$ & $\mathrm{MeOH}: \mathrm{H}_{2} \mathrm{O} 93: 7$ & 1 & 254 & $A$ and $B$ & [13] \\
\hline Lichrosorb RP-18 $(250 \times 4.6 \times 10)$ & $\mathrm{CH}_{3} \mathrm{CN}: \mathrm{H}_{2} \mathrm{O} 75: 25$ & 2.5 & 254 & $A$ and $B$ & [18] \\
\hline
\end{tabular}

${ }^{1}$ Guayulins C and D were quantified using a GC method; ${ }^{2}$ no further details were provided in the paper;

${ }^{3}$ in ref. [11], guayulins C and D were only identified, but not quantified, n.r. not reported in the original study.

Table 2. Chromatographic conditions proposed for the contemporary RP-HPLC separation and quantification of guayulins A, B, C and D in resin extracts from Guayule biomass.

\begin{tabular}{cc}
\hline Stationary Phase (Length, $\mathbf{m m} \times$ Diameter, $\mathbf{m m} \times$ Particle Size, $\mu \mathbf{m})$ & Ascentis $^{\circledR} \mathbf{C 1 8 ~}(\mathbf{2 5 0} \times \mathbf{4 . 6} \times \mathbf{5})$ \\
\hline Mobile phase $(v: v)$ & $\mathrm{CH}_{3} \mathrm{CN}: \mathrm{H}_{2} \mathrm{O} 80: 20$ \\
Elution mode & Isocratic \\
Volume $(\mu \mathrm{L})$ & 20 \\
Flux of mobile phase $\left(\mathrm{mL} \mathrm{min}{ }^{-1}\right)$ & 1.5 \\
UV wavelength $(\mathrm{nm})$ & 262 \\
Column temperature $\left({ }^{\circ} \mathrm{C}\right)$ & 25 \\
Length of the chromatographic run $(\mathrm{min})$ & 25 \\
\hline
\end{tabular}

The adoption of an Ascentis ${ }^{\circledR}$ C18 RP-HPLC column, more recent in comparison to those reported in literature (see Table 1) have allowed us to develop an isocratic method achieving the complete resolution at baseline level of all analytes in less than $20 \mathrm{~min}$, thus with large time savings of the chromatographic run as compared with the most recent literature method [11].

\subsection{Analysis of the Resin Extracts}

First, $2.5 \mathrm{~mL}$ of acetone (or ethanol) ASE extract of the resin from guayule biomass were evaporated, at room temperature and under reduced pressure, to dryness. The resin residue was dissolved in five milliliters of acetonitrile, and then filtered through a $0.45 \mu \mathrm{m}$ nylon filter before HPLC analysis. Figure 2 shows typical chromatograms of ASE resin extracts from Guayule leaves (Figure 2a) and coarse stems (Figure 2b).

Guayulins' peaks were identified by comparing retention times with those of standard solutions, and the attribution of each analyte was confirmed by spiking each peak in the real sample with a standard solution containing known amounts of pure guayulins. In order to determine the retention times, the reference standards were injected both individually and as a mixture. Quantification was 
accomplished by external linear calibration on three different concentration levels in the relevant linearity interval of each analyte. Each sample was analyzed three times, and each analytical datum is reported as the average value \pm the relevant standard deviation.
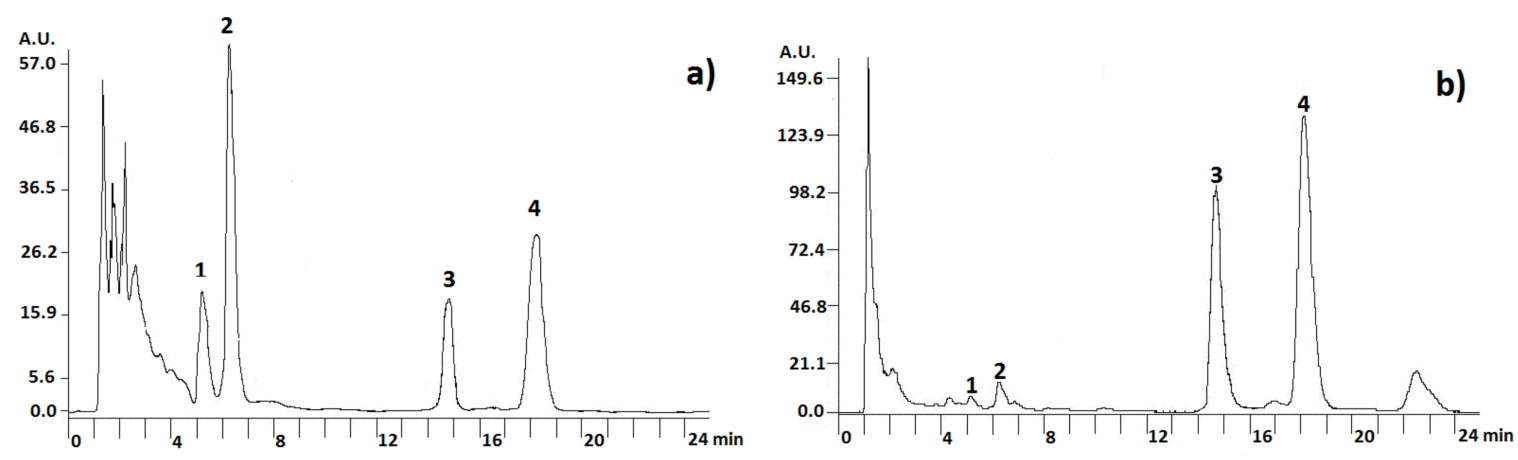

Figure 2. Guayulins A, B, C and D in resin extracts from (a) Guayule leaves (sample L1, extraction solvent: acetone) and (b) Guayule coarse stems (sample CS2, extraction solvent: ethanol). Peak attribution: 1-guayulin D; 2-guayulin C; 3-guayulin B; 4-guayulin A. A.U. = arbitrary units.

\subsection{Validation}

Table 3 lists the validation parameters for the method proposed. Low LoD values (always below $0.1 \mathrm{mg} \mathrm{L}^{-1}$ ) supported a good sensitivity of the method proposed, which is able to quantify analytes at concentration levels between 0.1 and $0.2 \mathrm{mg} \mathrm{L}^{-1}$, as a function of the analyte. Also, linearity is more than satisfactory. It has been checked only within the three orders of magnitude of concentration (i.e., inside the range of variability of the guayulins in the different parts of Guayule shrub), obtaining values of the determination coefficients $R^{2}$ ranging between 0.9994 and 0.9984 , but it is likely that the linear dynamic interval for each analyte may largely overcome these ranges. In addition, the analysis of the residuals of the regression line has excluded any possible deviation from linearity of each calibration plot. A preliminary evaluation of the samples has substantiated a very wide scattering of the concentration of the analytes in them. Due the fact that the meaning of the precision measurements crucially depends on analyte concentration [23], repeatability and intermediate precision were evaluated on the Guayule extracts characterized, for each analyte, by a concentration that was as close as possible to its average value in the relevant calibration interval. For this reason, the ethanol extract of sample FS1, the acetone extract of sample FS2, the acetone extract of sample FS1 and the ethanol extracts of sample CS1 were chosen for the precision measurements for guayulins A, B, C and D, respectively. The acceptability of the levels of the precision data, ranging between $1.3 \%$ and $3.2 \%$ (guayulin A) and $3.6 \%$ and $10 \%$ (guayulin D) for repeatability and intermediate precision, respectively, were evaluated by a fitness-for-purpose methodology based on the Horwitz's theory [23]. Operatively, acceptable values of the HorRat ratio (i.e., the ratio between the experimental CV and the theoretical CV calculated on the basis of Horwitz's theory) should be less than 1.5. Since the HorRat ratios for the procedure subject of this work never exceeded 0.6, the precision levels obtained from all analytes can be considered acceptable. Trueness evaluations have been accomplished with the same Guayule samples involved in the precision measurements. Recoveries obtained ranged between $73 \pm 5 \%$ (guayulin D) and $91 \pm 2 \%$ (guayulin B) for acetone extracts, whereas ethanol extracts gave recoveries between $77 \pm 8 \%$ (guayulin D) and $89 \pm 6 \%$ (guayulin A). The evaluation of the recovery values obtained on the basis of the AOAC guidelines [24] have allowed to substantiate, for all analytes, a slight underestimation bias. Unfortunately, the complete absence in the literature of validated methods for the determination of guayulins prevented us to make any comparison with results from previous studies. 
Table 3. Validation data.

\begin{tabular}{|c|c|c|c|c|c|c|c|c|c|c|}
\hline \multirow[t]{2}{*}{ Guayulin } & \multicolumn{2}{|c|}{ Sensitivity $(n=3)$} & \multicolumn{2}{|l|}{ Linearity } & \multicolumn{2}{|c|}{ Repeatability $(n=5)$} & \multicolumn{2}{|c|}{ Intermediate Precision $(n=5)$} & \multicolumn{2}{|c|}{ Bias $(n=2)$} \\
\hline & $\operatorname{LoD}\left(\mathrm{mg} \mathrm{L}^{-1}\right)$ & $\operatorname{LoQ}\left(\mathrm{mg} \mathrm{L}^{-1}\right)$ & $\begin{array}{c}\text { Concentration } \\
\text { Range }\left(\mathrm{mg} \mathrm{L}^{-1}\right)\end{array}$ & $\mathbf{R}^{2}$ & $\mathrm{CV}_{\text {exp, }}{ }^{\mathrm{a}}$ & HorRat $_{\mathrm{r}}{ }^{\text {b }}$ & $\mathrm{CV}_{\text {exp,IP }}{ }^{\mathrm{c}}$ & HorRat $_{\text {exp, IP }}{ }^{d}$ & $\begin{array}{l}\text { Recovery }\left(\% \pm S^{\mathrm{e}}\right) \\
\text { on Acetone }\end{array}$ & $\begin{array}{l}\text { Recovery }\left(\% \pm S D^{\mathrm{e}}\right) \\
\text { on Ethanol }\end{array}$ \\
\hline A & $0.032 \pm 0.004$ & $0.10 \pm 0.01$ & $0.2-500$ & 0.9994 & 1.3 & 0.26 & 3.2 & 0.43 & $87 \pm 4$ & $89 \pm 6$ \\
\hline B & $0.051 \pm 0.004$ & $0.15 \pm 0.01$ & $0.2-250$ & 0.9991 & 1.7 & 0.2 & 3.5 & 0.41 & $91 \pm 2$ & $88 \pm 8$ \\
\hline C & $0.034 \pm 0.009$ & $0.10 \pm 0.03$ & $0.1-200$ & 0.9989 & 1.5 & 0.58 & 4.0 & 0.39 & $81 \pm 9$ & $80 \pm 10$ \\
\hline D & $0.055 \pm 0.005$ & $0.16 \pm 0.02$ & $0.16-200$ & 0.9984 & 3.6 & 0.39 & 10 & 0.26 & $73 \pm 5$ & $77 \pm 8$ \\
\hline
\end{tabular}

${ }^{\text {a }} \mathrm{CV}_{\text {exp,r }}$ is the experimental coefficient of variation of repeatability, measured in the ethanol extract of FS1 for guayulin A, in the acetone extract of FS2 for guayulin B, in the acetone extract of FS1 for guayulin C and in the ethanol extract of CS1 for guayulin D, respectively; ${ }^{\mathrm{b}}$ HorRat $_{\mathrm{r}}$ is the ratio between $\mathrm{CV}_{\text {exp,r }}$ and the theoretical repeatability data $\left(\mathrm{CV}_{\mathrm{H}, \mathrm{r}}\right)$ according to Horwitz's theory; ${ }^{\mathrm{c}} \mathrm{CV}_{\mathrm{exp}, \mathrm{IP}}$ is the experimental coefficient of variation of intermediate precision, measured in the ethanol extract of FS1 for guayulin A, in the acetone extract of FS2 for guayulin B, in the acetone extract of FS1 for guayulin C and in the ethanol extract of CS1 for guayulin D, respectively;

${ }^{d}$ HorRat $I$ is is the ratio between $\mathrm{CV}_{\text {exp,IP }}$ and the theoretical intermediate precision data $\left(\mathrm{CV}_{\mathrm{H}, I \mathrm{P}}\right)$ according to Horwitz's theory; ${ }^{\mathrm{e}}$ standard deviation.

\subsection{Application on Real Samples}

Table 4 reports the analytical data obtained for the RP-HPLC determination of guayulins A, B, C and $\mathrm{D}$ in acetone and ethanol ASE extracts from different Guayule biomass (leaves (L samples), fine stems (FS samples) and coarse stems (CS samples)) harvested in January 2015 (dried samples, 1) and in September 2015 (fresh samples, 2).

Table 4. Concentration of guayulins A, B, C and D in acetone or ethanol extracts of Guayule biomass (leaves (L), fine stems (FS) and coarse stems (CS)) harvested in January 2015 (dried samples, 1) and in September 2015 (fresh samples, 2).

\begin{tabular}{|c|c|c|c|c|c|c|c|c|}
\hline \multirow[b]{2}{*}{ Sample (Solvent) } & \multicolumn{8}{|c|}{ Guayulins (mg kg $\left.{ }^{-1} \pm \mathrm{SD}\right)$} \\
\hline & A (A) & A (E) & B (A) & B (E) & C (A) & $C(E)$ & D (A) & D (E) \\
\hline L1 & $2080 \pm 40^{\mathrm{a}}$ & $2140 \pm 40^{\mathrm{a}}$ & $444 \pm 9^{a}$ & $449 \pm 4^{\mathrm{a}}$ & $1638 \pm 8^{\mathrm{a}}$ & $1400 \pm 45^{b}$ & $360 \pm 30^{a}$ & $330 \pm 30^{a}$ \\
\hline L2 & $2030 \pm 65^{a}$ & $2190 \pm 20^{a}$ & $428 \pm 6^{a}$ & $460 \pm 9^{a}$ & $1650 \pm 7^{a}$ & $1560 \pm 45^{b}$ & $370 \pm 20^{a}$ & $330 \pm 20^{a}$ \\
\hline FS2 & $4190 \pm 40^{\mathrm{a}}$ & $3640 \pm 10^{a}$ & $1190 \pm 20^{a}$ & $1150 \pm 9^{a}$ & $61.5 \pm 0.1^{a}$ & $26.4 \pm 0.7^{\mathrm{a}}$ & $<0.32 *$ & $<0.32 *$ \\
\hline CS1 & $8160 \pm 90^{a}$ & $9830 \pm 20^{a}$ & $700 \pm 10^{d}$ & $884.8 \pm 0.7^{\mathrm{a}}$ & $1010 \pm 4^{\mathrm{a}}$ & $1044 \pm 7^{\mathrm{a}}$ & $25 \pm 2^{a}$ & $55 \pm 2^{a}$ \\
\hline CS2 & $8800 \pm 100^{a}$ & $7800 \pm 100^{\mathrm{a}}$ & $3090 \pm 20^{a}$ & $2810 \pm 40^{\mathrm{a}}$ & $249 \pm 4^{\mathrm{a}}$ & $199 \pm 4^{\mathrm{a}}$ & $23 \pm 2^{a}$ & $16 \pm 2^{a}$ \\
\hline
\end{tabular}

SD = standard deviation; $n=3$; ${ }^{*}$ LoQ of guayulin D; paired values (i.e., same sample, same guayulins, different extraction solvent) followed by the same letter (from a to d) are not significantly different according to ANOVA test at $p=0.05$.

While data relative to guayulins $\mathrm{A}$ and $\mathrm{B}$ are roughly comparable with those previously reported in literature $[4,6,10,11]$, those relative to the amounts of guayulins $C$ and $D$ in acetone (or ethanol) extracts by different parts of the Guayule shrub are, to the best of our knowledge, unprecedented at all. Firstly, it is very important to highlight that these data are relative to a spontaneous Guayule shrub, whereas almost all literature data are relative to specific and well-known Guayule genotypes coming from cultivation.

\subsubsection{Guayulins in Guayule Leaves}

As a first remark, it is interesting to note that the relative composition in guayulins on leaves from the first and the second harvest is roughly constant, and this is probably due to the fact that the leaves of the shrubs harvested in September 2015 also arrived not entirely fresh in the laboratory, like those harvested in January 2015. The relative composition of both guayulins is almost the same as the nature of the solvent varies, with only the exception of a slight increase of the concentration of guayulins $\mathrm{C}$ and $\mathrm{D}$ and a faint decrease of the guayulin $\mathrm{A}$ in the acetone extract in comparison to those obtained by ethanol. The ratio between guayulin A and B in leaves is roughly close to 5:1. This data is significantly higher than that (i.e., 1.3) measured by Sidhu et al. for a Gila cultivar [10], but is within the range (between 2.5 and 7) measured by Teetor et al. [11] in brown leaves of three Guayule cultivars. On the other hand, leaves are very rich in guayulins $C$ and D. In particular, the amount of guayulin $\mathrm{D}$ in leaves is more than ten times higher than those found in the richest remaining part of Guayule 
shrub (i.e., the coarse stems from the January harvest, CS1), whereas the concentration of guayulin C in leaves is only ca. $50 \%$ higher than that measured in CS1. The significant increase of concentration of guayulins $\mathrm{C}$ and $\mathrm{D}$ is likely a consequence of the drying process of the guayule biomass. This fact confirms the hypotheses that these compounds could derive from oxidation/degradation pathways from guayulins A and B, respectively $[6,8]$. The increase of concentration is more evident for guayulin $C$ rather than for guayulin $D$, but it is possible that this could be caused by the difference between the relative abundance of guayulin A and guayulin B, respectively.

\subsubsection{Guayulins in Guayule Stems}

The highest amounts of guayulins A and B were found in the coarse stems (CS samples). Depending on the nature of the solvent used for the ASE extraction, amounts ranging between $7800 \pm 100$ and $9830 \pm 20 \mathrm{mg} \mathrm{kg}^{-1}$ were measured for guayulin A, whereas concentrations ranging between $700 \pm 10$ and $3090 \pm 20 \mathrm{mg} \mathrm{kg}^{-1}$ were measured for guayulin $\mathrm{B}$. These concentrations are in partial agreement with those observed by Teetor et al. for guayulin A [11] and by Sidhu et al. for guayulin B [10]. Indeed, the concentrations of guayulin B measured by Teetor et al. [11] and the amounts of guayulin A found by Sidhu et al. [10] were not as high as those measured in this study. It is interesting to note that the concentration ratio between guayulin A and guayulin B, which is roughly constant at varying of the nature of the solvent, increases by more than $400 \%$ passing from fresh to aged stems, and this is due to the loss of more than $70 \%$ in the average amount of guayulin $B$ during the storage. On the other hand, data show that changes of the concentration of the guayulin A, albeit much lower than those shown by guayulin B, depend by both the nature of the extraction solvent and the aging of the sample in non-trivial way. Furthermore, the amount of guayulin $C$ in these samples tends to sharply increase (up to five times higher) with ageing, whereas the concentration of this analyte is invariant towards the nature of the extraction solvent. Finally, only a few tens of $\mathrm{mg}$ $\mathrm{kg}^{-1}$ of guayulin $\mathrm{D}$ were found in the coarse stem samples. A moderate increase in concentration of this analyte, likely due to aging effects, was observed only in ethanol extracts.

The amounts of guayulins in fine stems are intermediate between those measured in coarse stems and leaves. The amounts of guayulins A and B in these samples are normally only $30-50 \%$ of the relevant values measured for coarse stems, whereas the loss of guayulin B during the ageing is only between $50 \%$ and $55 \%$ the amount measured in fresh samples. On the other hand, very high increases of concentration of guayulin C were found (ranging between $600 \%$ and $770 \%$ in samples extracted with acetone and ethanol, respectively) passing from fresh to aged samples, whereas just a few $\mathrm{mg} \mathrm{kg}^{-1}$ of guayulin D were measured only in FS1 samples, being the remaining samples below the relevant LoQ for this analyte.

\section{Conclusions}

To the best of our knowledge, for the first time a RP-HPLC method specifically aimed to simultaneously measure the concentration of the known guayulins in resin extracts from parts of Guayule shrub has been developed, validated and applied to real samples. The development of the method has first involved the optimization of the resin extraction procedure from the Guayule biomass among three different approaches (warm solvent extraction, ultrasonic assisted extraction, accelerated solvent extraction). The latter technique provided the best performances and was chosen to be used in the analytical method. The HPLC separation was accomplished by means of an isocratic elution on an Ascentis ${ }^{\circledR}$ C18 column with an 80:20 (v:v) mixture of acetonitrile and water. In this way, the separation at the baseline level of all analytes in less than twenty minutes was obtained. Low LoD and LoQ values, a very good linearity over more than three orders of magnitude and a very good precision characterized the proposed method. On the other hand, a slight underestimation bias was observed in all analytes. The method has been successfully tested to fresh and aged samples of spontaneous Guayule shrub, harvested in the Chihuhaua desert (Laredo, Mexico). The guayuline amount was measured as a function of the ageing level of the sample (fresh or air-exposed for eight months), the 
parts of the plant (leaves, fine stems, coarse stems) and the nature of the extraction solvent (acetone or ethanol). The data obtained are in substantial agreement with those described in the literature for guayulins A and B, while there are no reference data for guayulins $C$ and $D$. The analysis of the stems revealed evident differences in the concentration of guayulins as a function of their diameter, of the ageing level and, to a lesser extent, of the nature of the extraction solvent. On the other hand, the analysis of the leaves showed no meaningful differences among data obtained from samples obtained in the two crops or by variation of the solvent nature. The leaves were the richest fractions in guayulines $C$ and $D$, while the coarse stems were the richest in guayulines $A$ and $B$. The obtained data showed that leaves constitute the fraction of Guayule that is probably most is prone to the effect of oxidative degradation of guayulins A (and B) and the consequent transformation in guayulins $C$ (and D). This behavior is much less evident, where it is present, on the stems. This analytical method is expected to be useful in any further qualitative and quantitative evaluation of guayulins in different parts of Guayule shrub as a function of the nature of the genotype, or the adoption of different cultivation practices.

Acknowledgments: This work was financed by the Italian “Ministero dell'Istruzione, dell'Università e della Ricerca" (CTN-001-00063-46446 ALBE BIOMASSE Lignocellulosiche alternative per lo sviluppo di elastomeri).

Author Contributions: N.S., G.S. and A.M. conceived and designed the experiments; P.M. and I.I. performed the experiments; M.I.P., V.M.N. and J.I.L. analyzed the data; E.R. and A.O.-E. provided Guayule samples; G.S. and N.S. wrote the paper.

Conflicts of Interest: The authors declare no conflict of interest.

\section{References}

1. McIntyre, D.; Stephens, H.L.; Schloman, W.W., Jr.; Bhowmick, A.K. Guayule rubber. In Handbook of Elastomers, 2nd ed.; Bhowmick, A.K., Stephens, H., Eds.; CRC Press: Boca Raton, FL, USA, 2000; ISBN 9780824703837.

2. Van Beilen, J.B.; Poirier, Y. Establishment of new crops for the production of natural rubber. Trends Biotechnol. 2007, 25, 522-529. [CrossRef] [PubMed]

3. Van Beilen, J.B.; Poirier, Y. Guayule and Russian Dandelion as Alternative Sources of Natural Rubber. Crit. Rev. Biotechnol. 2007, 27, 217-231. [CrossRef] [PubMed]

4. Schloman, W.W., Jr.; Garrot, D.J., Jr.; Ray, D.T.; Bennett, D.J. Seasonal Effects on Guayule Resin Composition. J. Agric. Food Chem. 1986, 34, 177-179. [CrossRef]

5. Eagle, F.A. Guayule. Rubber Chem. Technol. 1981, 54, 662-684. [CrossRef]

6. Schloman, W.W., Jr.; Hively, R.A.; Krishen, A.; Andrews, A.M. Guayule Byproduct Evaluation: Extract Characterization. J. Agric. Food Chem. 1983, 31, 873-876. [CrossRef]

7. Romo, J.; Romo De Vivar, A.; Ortega, A.; Diaz, E. Guayulins A and B, new sesquiterpenes isolated from guayule. Rev. Latinam. Quim. 1970, 1, 132-135.

8. Martinez, M.; Flores, G.; Romo de Vivar, A.; Reynolds, G.; Rodriguez, E. Guayulins C and D from Guayule (Parthenium Argentatum). J. Nat. Prod. 1986, 49, 1102-1103. [CrossRef] [PubMed]

9. Watkins, S.F.; Fronczek, F.R.; Chiari, G.; Reynolds, G.W.; Rodriguez, E. Molecular structure of guayulin A. J. Nat. Prod. 1985, 48, 631-633. [CrossRef] [PubMed]

10. Sidhu, O.P.; Ratti, N.; Behl, H.M. Quantitative and Qualitative Variations in Resin Content and Guayulins (A and B) among Different Guayule Cultivars. J. Agric. Food Chem. 1995, 43, 2012-2015. [CrossRef]

11. Teetor, V.H.; Ray, D.T.; Schloman, W.W., Jr. Evaluating chemical indices of guayule rubber content: Guayulins A and B. Ind. Crop. Prod. 2009, 29, 590-598. [CrossRef]

12. Mau, C.; Cornish, K. Guayule Plants, Products, and Derivatives. US Patent 8013213, 6 September 2011.

13. Mohan Behl, H.; Marchand, B.; Rodriguez, E. Inheritance of Sesquiterpenoid Phenolic Acid Esters (Guayulins) in $\mathrm{F}_{1}$ Hybrids of Parthenium (Asteraceae). Z. Naturforsch. 1983, 38, 494-496. [CrossRef]

14. Rodriguez, E.; Towers, G.H.N.; Mitchell, J.C. Biological activities of sesquiterpene lactones. Phytochemistry 1976, 15, 1573-1580. [CrossRef]

15. Rodriguez, E.; Reynolds, G.W.; Thompson, J.A. Potent Contact Allergen in the Rubber Plant Guayule (Parthenium argentatum). Science 1981, 211, 1444-1445. [CrossRef] [PubMed] 
16. Cornish, K.; Williams, J.L.; Kirk, M.; Teetor, V.H. Evaluation \& control of potential sensitizing \& irritating chemical components in natural rubber latex extracted from the industrial crop guayule. Ind. Biotechnol. 2009, 5, 245-252. [CrossRef]

17. Coffelt, T.A.; Nakayama, F.S.; Ray, D.T.; Cornish, K.; McMahan, C.M. Post-harvest storage effects on guayule latex, rubber, and resin contents and yields. Ind. Crop. Prod. 2009, 29, 326-335. [CrossRef]

18. Proksch, P.; Mohan Behl, H.; Rodriguez, E. Detection and quantification of guayulins A and B in Parthenium argentaturn (guayule) and $\mathrm{F}_{1}$ hybrids by high-performance liquid chromatography. J. Chromatogr. A 1981, 213, 345-348. [CrossRef]

19. Ray, D.T.; Veatch-Blohm, M.E.; Teetor, V.H.; Walsh, B. Upper and Lower Heritability Estimates in Guayule Based on Mode of Reproduction. J. Am. Soc. Hort. Sci. 2007, 132, 213-218.

20. Lloyd, F.E. Guayule (Parthenium Argentatum Gray), a Rubber Plant of the Chihuahuan Desert; Carnegie Institution of Washington: Washington, DC, USA, 1911; pp. 57-58.

21. Norma Oficial Mexicana NOM-007-RECNAT-1997. Procedimientos, Criterios y Especificaciones Para Realizar el Aprovechamiento, Transporte y Almacenamiento de Ramas, Hojas o Pencas, Flores, Frutos y Semillas; Diario Oficial de la Federaciòn de los Estados Unidos Mexicanos: Ciudad de Mexico, Mexico, 1997.

22. Mocak, J.; Bond, A.M.; Mitchell, S.; Schollary, G. A statistical overview of standard (IUPAC and ACS) and new procedures for determining the limits of detection and quantification: Application to voltammetric and stripping techniques. Pure Appl. Chem. 1997, 69, 297-328. [CrossRef]

23. Horwitz, W. Evaluation of analytical methods used for regulation of foods and drugs. Anal. Chem. 1982, 54, 67A-76A. [CrossRef]

24. AOAC International. AOAC Peer Verified Methods Programs, Manual on Policies and Procedures; AOAC International: Arlington, VA, USA, 1998.

25. Salvucci, M.E.; Coffelt, T.A.; Cornish, K. Improved methods for extraction and quantification of resin and rubber from guayule. Ind. Crop. Prod. 2009, 30, 9-16. [CrossRef]

26. Yeom, H.; Suh, J.H.; Youm, J.R.; Han, S.B. Simultaneous Determination of Triterpenoid Saponins from Pulsatilla koreana using High Performance Liquid Chromatography Coupled with a Charged Aerosol Detector (HPLC-CAD). Bull. Korean. Chem. Soc. 2010, 31, 1159-1164. [CrossRef] 